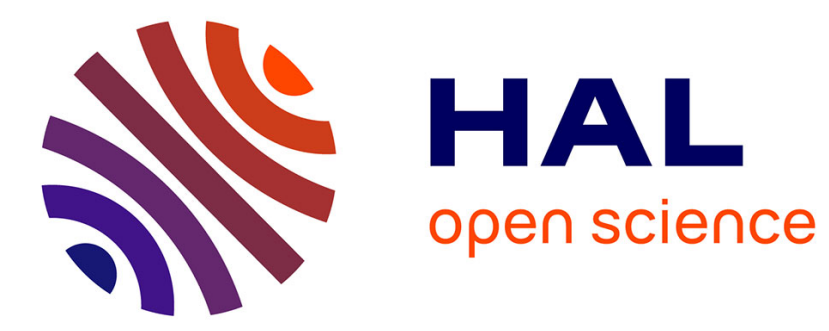

\title{
Localised bending modes in split ring resonators
}

Sébastien Guenneau, Alexander Movchan, Natasha Movchan

\section{To cite this version:}

Sébastien Guenneau, Alexander Movchan, Natasha Movchan. Localised bending modes in split ring resonators. Physica B: Condensed Matter, 2007, 394 (2), pp.141-144. hal-00186678

\section{HAL Id: hal-00186678 \\ https://hal.science/hal-00186678}

Submitted on 3 Dec 2007

HAL is a multi-disciplinary open access archive for the deposit and dissemination of scientific research documents, whether they are published or not. The documents may come from teaching and research institutions in France or abroad, or from public or private research centers.
L'archive ouverte pluridisciplinaire HAL, est destinée au dépôt et à la diffusion de documents scientifiques de niveau recherche, publiés ou non, émanant des établissements d'enseignement et de recherche français ou étrangers, des laboratoires publics ou privés. 


\title{
Localised bending modes in split ring resonators
}

\author{
S. Guenneau, ${ }^{\mathrm{a}, \mathrm{b}}$ A.B. Movchan, ${ }^{\mathrm{a}}$ N.V. Movchan ${ }^{\mathrm{a}}$ \\ a Department of Mathematical Sciences, $M$ \& $O$ Building, \\ Peach Street, Liverpool L69 3BX, United Kingdom \\ b Institut Fresnel, UMR CNRS 6133, University Aix-Marseille III, case 162, F13397 Marseille Cedex 20, France
}

\begin{abstract}
We present band diagrams for in-plane elastic waves propagating within a doubly periodic structure involving Split Ring Resonators. From the Navier equations we derive that the lowest resonant frequencies are associated with localised bending modes solutions of a fourth order differential equation in thin bridges, which are responsible for the appearance of a low frequency stop band. Potential applications lie in the design of earthquake resistant systems.
\end{abstract}

Key words: Thin-bridges, elastic split ring resonators, locally resonant structures PACS: 47.11.St, 42.15.-i, 78.20.Bh

\section{Introduction}

The elastic materials considered here include locally resonant elements known as Split Ring Resonators (SRR). SRR were originally used as building blocks for periodic materials exhibiting artificial magnetism by Pendry and his coauthors [1]. In [2] an asymptotic algorithm was proposed to characterize the resonant frequencies of SSR in the case of anti-plane shear waves. An improved asymptotic estimate was derived for the analogous case of transverse electric waves in [3]. Here, we broaden the analysis of [2] to the vector case of in-plane elastic waves.

\section{Set up of the spectral problem}

We consider a doubly periodic array of 'defects' (see Figure 1) embedded in an elementary cell $Y=[0 ; 1] \times[0 ; 1]$ repeated periodically in the $x_{1} x_{2}$-plane. In-plane propagating waves are modelled by a displacement field $\mathbf{U}\left(x_{1}, x_{2}, t\right)$ which satisfies the Navier equation

$\mu \Delta \mathbf{U}+(\lambda+\mu) \nabla \nabla \cdot \mathbf{U}-\rho \frac{\partial^{2}}{\partial t^{2}} \mathbf{U}=0$

in $Y \backslash \bigcup_{j} \overline{\Omega_{j}}$. Assuming a time-harmonic dependence $\mathbf{U}=$ $\mathbf{u}\left(x_{1}, x_{2}\right) \exp (i \omega t)$, we obtain the Navier equation in the form

$\mu \Delta \mathbf{u}+(\lambda+\mu) \nabla \nabla \cdot \mathbf{u}+\rho \omega^{2} \mathbf{u}=0$
Here, $\mathbf{u}$ represents the amplitude of the displacement vector field within an elastic medium with the mass density $\rho$ and the Lamé elastic moduli $\lambda$ and $\mu ; \omega$ stands for the radian frequency of vibration. We also assume that $\mathbf{u}$ satisfies traction free boundary conditions on the contours of defects i.e.

$\sigma_{i j} n_{j}=0, i, j=1,2$,

where $\mathbf{n}$ denotes the outward unit normal to the contours, and $\sigma_{i j}$ are components of the stress tensor.

Finally, u should satisfy the Floquet-Bloch condition

$\mathbf{u}\left(\mathbf{x}+m \mathbf{e}^{1}+n \mathbf{e}^{2}\right)=\mathbf{u}(\mathbf{x}) e^{i\left(k_{1} m+k_{2} n\right)}$,

within the doubly periodic array. Here $m$ and $n$ are integers, and $k_{1}, k_{2}$ represent the components of the Floquet-Bloch vector $\mathbf{k}$; $\mathbf{e}^{j}$ are the unit basis vectors of the Cartesian coordinate system in $\mathbb{R}^{2}$.

Let us note that in-plane dilatational and shear waves propagate at different velocities, namely $v_{p}=\sqrt{(\lambda+2 \mu) / \rho}$ and $v_{s}=\sqrt{\mu / \rho}$. We would like to consider a particular case when the defect $\Omega$ in $Y$ is of the shape of the letter $C$ (see Figure 1). Formally, $\Omega=\{a<|\mathbf{x}|<b\} \backslash \overline{\Pi_{\varepsilon}}$, where $a$ and $b$ are given constants and $\Pi_{\varepsilon}$ is a thin ligament between the 'ends of the letter $C$ ':

$\Pi_{\varepsilon}=\left\{\left(x_{1}, x_{2}\right): a<x_{1}<b,\left|x_{2}\right|<\varepsilon h / 2\right\}$.

In (5), $h$ is a given constant, and $\varepsilon$ is a small positive nondimensional parameter. 


\section{Asymptotic algorithm for an elastic multi-structure}

To proceed with the asymptotic algorithm, we first assume that the longitudinal displacement $U_{1}=u_{1} \exp (i \omega t)$ whereas the transverse displacement $U_{2}=u_{2} \exp (i \omega \epsilon t)$ in (1): transverse vibrations $u_{2}$ (bending modes) occur at low frequencies. This amounts to introducing a scaled time variable $T=\varepsilon t$. Let us also introduce the scaled spatial variable $\xi=x_{2} / \varepsilon$. Altogether, the rescaled Navier equation deduced from (1) is

$$
\begin{aligned}
& \mu\left(\frac{1}{\varepsilon^{2}} \frac{\partial^{2}}{\partial \xi^{2}}+\frac{\partial^{2}}{\partial x_{1}^{2}}\right)\left(\begin{array}{l}
u_{1} \\
u_{2}
\end{array}\right)+(\lambda+\mu) \\
& \left(\begin{array}{ll}
\frac{\partial^{2}}{\partial x_{1}^{2}} & \frac{1}{\varepsilon} \frac{\partial^{2}}{\partial x_{1} \partial \xi} \\
\frac{1}{\varepsilon} \frac{\partial^{2}}{\partial x_{1} \partial \xi} & \frac{1}{\varepsilon^{2}} \frac{\partial^{2}}{\partial \xi^{2}}
\end{array}\right)\left(\begin{array}{l}
u_{1} \\
u_{2}
\end{array}\right)=-\rho \omega^{2}\left(\begin{array}{l}
u_{1} \\
\varepsilon^{2} u_{2}
\end{array}\right),
\end{aligned}
$$

in $\Pi_{1}=\left\{\left(x_{1}, \xi_{2}\right): a<x_{1}<b,\left|\xi_{2}\right|<h / 2\right\}$.

On the upper and lower boundaries of $\Pi_{\varepsilon},(3)$ reduces to

$\lambda \delta_{i 2} \sum_{k=1}^{2} \frac{\partial u_{k}}{\partial x_{k}}+\mu\left(\frac{\partial u_{i}}{\partial x_{2}}+\frac{\partial u_{2}}{\partial x_{i}}\right)=0$, for $i=1,2$,

where $\delta_{i j}$ is the Kronecker symbol which takes the value 1 if $i=j$ and 0 otherwise.

The rescaled traction free boundary conditions on $\Pi_{1}$ deduced from (7) are

$\frac{1}{\varepsilon}\left(\begin{array}{ll}\mu \frac{\partial}{\partial \xi} & 0 \\ 0 & (\lambda+2 \mu) \frac{\partial}{\partial \xi}\end{array}\right) \mathbf{u}+\left(\begin{array}{ll}0 & \mu \frac{\partial}{\partial x_{1}} \\ \lambda \frac{\partial}{\partial x_{1}} & 0\end{array}\right) \mathbf{u}=\mathbf{0}$.

It can be shown (see for instance [4]) that the displacement $\mathbf{u}$ admits the asymptotic form

$$
\begin{gathered}
\mathbf{u} \sim \varepsilon^{-2} \mathbf{u}^{(I I, 0,0)}+\varepsilon^{-1} \mathbf{u}^{(I I, 0,1)}+\left(\mathbf{u}^{(I, 0,0)}+\mathbf{u}^{(I I, 0,2)}\right) \\
+\varepsilon\left(\mathbf{u}^{(I, 0,1)}+\mathbf{u}^{(I I, 0,3)}\right)+\varepsilon^{2} \mathbf{U}^{(0)} .
\end{gathered}
$$

We then collect terms of same powers of $\varepsilon^{-k}$ in (6) and $\varepsilon^{-k+1}$ in (8).

\section{A fourth-order differential equation for localised bending modes}

Taking $k=2$, we derive that $\mathbf{u}^{(I, 0,0)}=\left(u_{1}^{(0)}\left(x_{1}\right), 0\right)^{T}$ (it is $\xi$ independent and has zero transverse component). These longitudinal modes are solutions of a second-order differential operator

$$
\frac{4 \mu(\lambda+\mu)}{(\lambda+2 \mu)} \frac{d^{2} u_{1}^{(0)}}{d x_{1}^{2}}+\omega^{2} \rho u_{1}^{(0)}=0, a<x_{1}<b .
$$

For $k=4$, we derive that $\mathbf{u}^{(I I, 0,0)}=\left(0, u_{2}^{(0)}\left(x_{1}\right)\right)^{T}$ where the transverse modes $u_{2}^{(0)}\left(x_{1}\right)$ satisfy a fourth-order differential equation corresponding to low-frequency bending modes

$\frac{h^{2} \mu(\lambda+\mu)}{3(\lambda+2 \mu)} \frac{d^{4} u_{2}^{(0)}}{d x_{1}^{4}}-\omega^{2} \rho u_{2}^{(0)}=0, a<x_{1}<b$.

The two ordinary differential equations (10) and (11) are supplied with boundary conditions at the junction point $x_{1}=a$ connecting the thin-bridge $\Pi_{\varepsilon}$ to the large body $\Sigma$ (see for instance [4]) and at the point $x_{1}=b$. For longitudinal modes, the boundary conditions are $u_{1}^{(0)}(a)=A$ and $u_{1}^{(0)}(b)=0$ as explained in [2] and using Newton's second law we find

$\omega^{2} \sim \frac{\mu \varepsilon h}{M(b-a)} \frac{4(\lambda+\mu)}{(\lambda+2 \mu)}$.

For pure bending modes, (11) is complemented by

$u_{2}^{(0)}(a)=A, u_{2}^{\prime(0)}(a)=0, u_{2}^{(0)}(b)=0, u_{2}^{\prime(0)}(b)=0$.

When $\omega \ll 1$, we deduce from (11) and (13) that $u_{2}^{(0)} \sim$ $\left(3 A /(b-a)^{2}\right) x_{1}^{2}-\left(2 A /(b-a)^{3}\right) x_{1}^{3}$. Furthermore, at the junction $a$, we also have

$-M \omega^{2} A=\frac{\varepsilon^{3} h^{3} \mu(\lambda+\mu)}{3(\lambda+2 \mu)} \frac{d^{3} u_{2}^{(0)}}{d x_{1}^{3}}(a)$.

We finally obtain

$\omega^{2} \sim \frac{\mu \varepsilon^{3} h^{3}}{M(b-a)^{3}} \frac{(\lambda+\mu)}{3(\lambda+2 \mu)}$.

Of course, this estimate changes for rotational bending modes. Full details of the derivation are provided in [5].

\section{Discussion of numerical results and perspectives}

We then investigate numerically stop band properties for in-plane elastic waves in arrays of SRR. This was done using finite elements (implementing the weak form of (2)(4)). We will see that the resonant modes of a single SRR discussed in the previous section are associated with the presence of a low frequency band gap for bending modes.

In what follows, we used normalised elastic parameters $\lambda=2.3, \mu=1, \rho=1$.

We give in Figure 1(a) the band diagram for a square array of pitch $d$ of voids of radius $0.43 d$ showing a full phononic band gap for the range of normalised frequencies $[4.35,5.05]$. The geometry of the array and the first Brillouin zone $\Gamma M X$ are depicted in Figure 1(b). In Figure 1(c) we depict the band diagram for a square array of SRR of inner radius $0.3 d$ and outer radius $0.43 d$ with a thin ligament of thickness $0.05 d$ and length $0.13 d$, showing a full phononic band gap for the range of normalised frequencies $\omega d / v_{s} \in$ $[3.95,4.80]$. The associated multistructure $\Sigma \cup \Pi_{\varepsilon}$ is drawn 

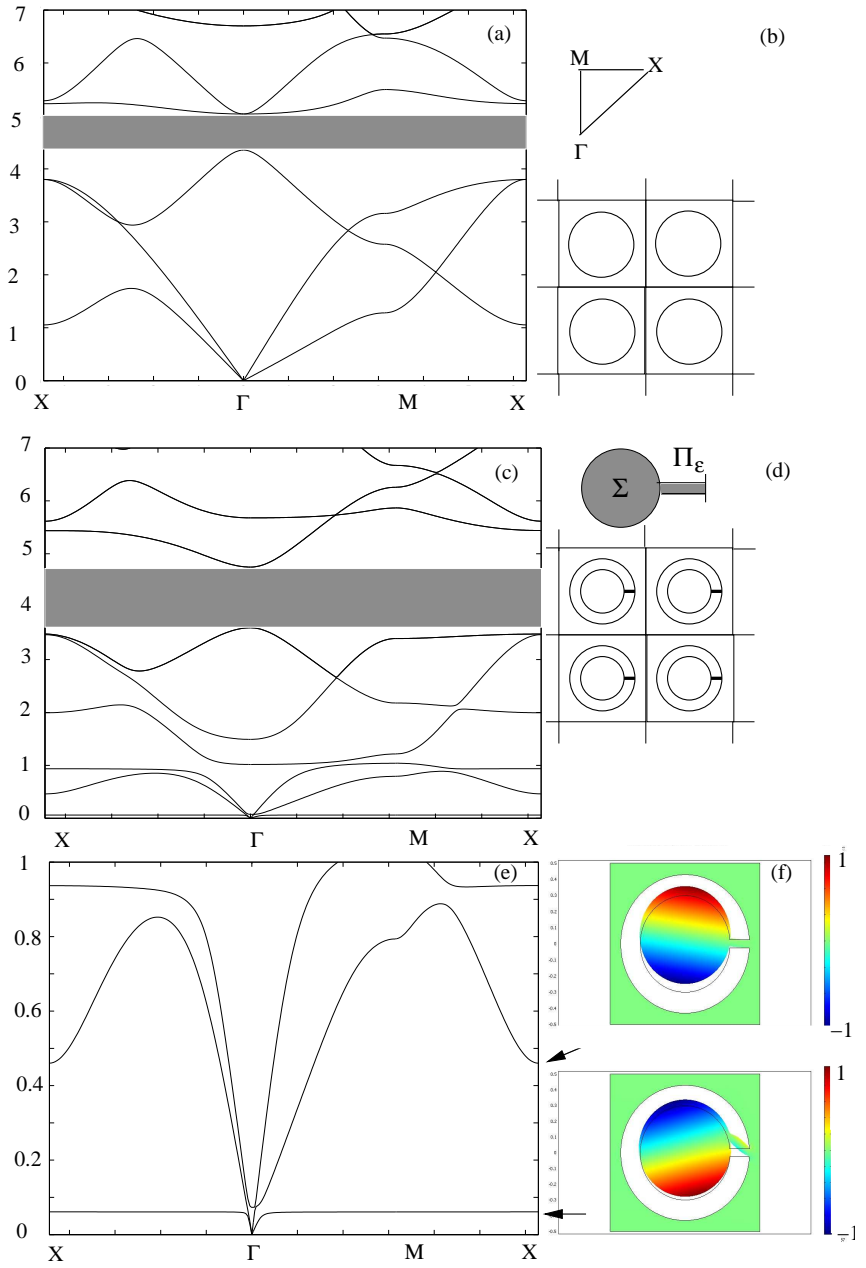

Fig. 1. (a) Band diagram for a square array of pitch $d$ of voids of radius $0.43 d$; (b) Array and first Brillouin zone $\Gamma M X$; (c) Band diagram for a square array of SRR of inner radius $0.3 d$ and outer radius $0.43 d$. (d) Multistructure $\Sigma \cup \Pi_{\varepsilon}$; (e) Zoom of band diagram (c) for low frequencies; (f) First two localised eigenmodes.

in Figure 1(d). We then have a closer look at the low frequency bands and zoom the band diagram of Figure 1(c) in order to magnify the low frequency stop band for bending modes in the frequency range $\omega d / v_{s} \in[0.075,0.083]$, where $v_{s}$ is the velocity of shear waves (see Figure 1(e)). This gap is associated with a pure bending mode which has a frequency $\omega d / v_{s}=0.075$ at point $X$ of the first Brillouin zone (see Figure 1(f)). Its frequency compares well with the asymptotic estimate: the normalised frequency for pure bending modes is $\omega d / v_{s} \sim 0.0697$ (about 7 per cent of inaccuracy). We notice that these frequencies are very low, hence this inaccuracy between the finite element result and the asymptotic estimate is really acceptable. For longitudinal modes, the asymptotic estimate gives $\omega d / v_{s} \sim 1.947$. We note that this estimate corresponds to the fourth curve on the band diagram of Figure 1(c), which has a frequency $\omega d / v_{s}=1.975$ at point $X$ of the first Brillouin zone (which means less than 1.5 per cent of inaccuracy for the asymptotic estimate). A rotational bending mode is also present at frequency $\omega d / v_{s}=0.43$ on the edge of the Brillouin zone and it is depicted in Figure 1(f)).

The SRR are clearly responsible for the appearance of a full band gap for one wave polarisation in the subwavelength regime (the pitch of the array is typically onehundredth of the wavelength). Hence, our study suggests that potential applications of elastic SRR lie in earthquake resistant systems or elastic waves polarisers. Our study may also pave the way towards the realisation of elastic phononic superlenses.

\section{References}

[1] Pendry, J.B., Holden, A.J., Roberts, D.J. and Stewart, W.J. IEEE Trans. Microwave Theory Tech. 47, (1999) 2075.

[2] Movchan, A.B. and Guenneau, S., Phys. Rev. B 70, (2004) 125116.

[3] Movchan, N.V., Guenneau, S., Movchan A.B. and McPhedran, R.C., Physica B (accepted).

[4] V. Kozlov, V. Maz'ya and A.B. Movchan, Fields in multistructures. Asymptotic analysis, Oxford University Press, 1999.

[5] S. Guenneau, A.B. Movchan and N.V. Movchan Waves Rand. Comp. Med. (submitted). 